\title{
Calendario de vacunación en los nuevos médicos residentes procedentes de Perú: Hospital Universitario Ramón y Cajal
}

\author{
Vaccine schedule in new resident physicians from Peru: Ramon y Cajal University \\ Hospital
}

\author{
Gian C. Navarro Chumbes, Marina Fernández Escribano, Ignacio Sánchez-Arcilla Conejo. \\ Servicio de Prevención de Riesgos Laborales, Hospital Universitario Ramón y Cajal, Madrid. España. \\ Recibido: 11-11-10 \\ Aceptado: 15-02-11

\section{Correspondencia} \\ Gian C. Navarro Chumbes \\ Servicio de Prevención de Riesgos Laborales. \\ Hospital Universitario Ramón y Cajal. \\ Carretera de Colmenar Viejo, Km 9, 100. 28049 Madrid. \\ E-mail: gcnavarro@yahoo.com / gcnavarro@hotmail.com
}

Resumen

Introducción: En los últimos años España se ha convertido en un país receptor de inmigrantes y el personal sanitario no es una excepción. Al momento de valorar el estado vacunal de los médicos residentes de inicio es importante tener en cuenta su procedencia.

Objetivo: Realizar una revisión sobre la vacunación existente en el Perú, antecedentes y seroprevalencia de las patologías inmunoprevenibles en el personal sanitario peruano; y en base a lo encontrado determinar las pautas de vacunación a seguir en caso no se aporte documentación de vacunación previa para aquellos médicos residentes de inicio que provengan del Perú.

Material y Métodos: Revisión bibliográfica.

Resultados: Los datos encontrados sugieren que no existe evidencia que nos indique una correcta pauta de vacunación en los médicos residentes que proceden de Perú.

Conclusiones: A todo médico residente de inicio proveniente de Perú que no aporte cartilla de vacunación se le procederá a vacunar como si fuese un adulto no vacunado, siempre teniendo en cuenta que si existe documentación de dosis previas se completarán las pautas sin reiniciar o repetir las dosis.

Med Segur Trab (Internet) 2011; 57 (222) 23-33

Palabras clave: Vacunación, Residente hospitalario, Inmigración, Perú.

\section{Abstract}

Introduction: In recent years, Spain has been receiving immigrants of all working sectors and health staff is not an exception. When evaluating a vaccine schedule of first year resident physicians, it is important to know where they are arriving from.

Objective: Make a review about vaccination in Peru, background and seroprevalence of immunopreventable pathology in Peruvian health staff; and bearing in mind the information, determine a 
vaccine schedule for first year resident physicians from Peru whose information is not available because they do not have previous vaccination documents.

Method and materials: We made a bibliographic review.

Results: The information obtained suggests that there is no evidence of correct vaccination in first year resident physicians from Peru.

Conclusions: All first year resident physicians from Peru who do not have vaccination documents will be vaccinated as if they were a non vaccinated adult. We will always bear in mind that if there is any document about previous doses, we will continue without beginning or repeating doses.

Med Segur Trab (Internet) 2011; 57 (222) 23-33

Key words: Vaccination, Hospital resident, Immigration, Peru. 


\section{INTRODUCCIÓN}

España se ha convertido en los últimos años en un país receptor de inmigrantes, igualándose en estos últimos años, la cifra de inmigrantes a la del resto de los países de la Unión Europea $^{2}$, por lo que un aspecto muy relevante a tener en cuenta es la procedencia de la población inmigrante y en este sentido su procedencia varía en las diferentes Comunidades Autónomas (CCAA) a predominio de los núcleos urbanos; asimismo es imprescindible considerar la procedencia a la hora de plantear estrategias de vacunación ya que al conocer el país de procedencia y los calendarios de vacunación que allí se aplican entonces podremos desarrollar una estrategia de vacunación mucho más eficaz.

El personal sanitario se ve afectado principalmente por el riesgo biológico por lo que los programas de vacunación tienen una gran importancia para el control de este riesgo $^{3}$. Las vacunas recomendadas como prioritarias en el personal sanitario para los centros de control y prevención de enfermedades americano (Centres for Disease Control and Prevention, CDC) y el comité asesor de prácticas de inmunización (Advisory Committee on Immunization Practices, ACIP) son: paperas, rubéola, sarampión, varicela, gripe y hepatitis B como prioritarias, y hepatitis A, meningococo, tos ferina, fiebre tifoidea en segundo lugar, según factores ${ }^{4}$.

El personal sanitario no se ve ajeno a este tipo de fenómenos migratorios; se ha observado que en los últimos años la formación de médicos especialistas está siendo ocupada, en un porcentaje que va en aumento, por médicos extracomunitarios; en el año 2010 se encontró que de las 8000 plazas ofertadas el 45\% fueron extracomunitarios, y se calcula que el año que viene el porcentaje será de $50 \%$.

De acuerdo a los datos obtenidos del Instituto Nacional de Estadísticas (INE) el número de iberoamericanos con autorización de estancia por estudios en territorio español durante el periodo 2005-2008 ha ido en aumento, tal y como se observa en la Tabla I.

Tabla I. Iberoamericanos con autorización de estancia por estudios en territorio español periodo 2005-2008

\begin{tabular}{lrrrrrrrrrrrr}
\hline & \multicolumn{3}{c}{2005} & \multicolumn{3}{c}{2006} & & & 2007 & & \multicolumn{2}{c}{2008} \\
& Total & VAA & VPA & Total & VAA & VPA & Total & VAA & VPA & Total & VAA & VPA \\
\hline Ambos sexos & 19789 & -3213 & $-13,97$ & 21264 & 1475 & 7,45 & 25368 & 4104 & 19,3 & 25977 & 609 & 2,4 \\
Varones & 9694 & -1237 & $-11,32$ & 10235 & 541 & 5,58 & 11899 & 1664 & 16,26 & 12049 & 150 & 1,26 \\
Mujeres & 10095 & -1973 & $-16,35$ & 11029 & 934 & 9,25 & 13469 & 2440 & 22,12 & 13924 & 455 & 3,38 \\
\hline
\end{tabular}

VAA: Variación absoluta anual.

VPA: Variación porcentual anual.

Unidades: número de personas.

Fuente:Anuario de Estadísticas del Ministerio de Trabajo e Inmigración.

El Hospital Universitario Ramón y Cajal (HURC) no es una excepción a este fenómeno migratorio, ya que anualmente se aprecia que en las diversas especialidades que existe en el hospital hay médicos residentes extranjeros, y de acuerdo a la Encuesta Inicial de Valoración de Salud realizado a los médicos residentes de primer año que lleva a cabo anualmente el Servicio de Prevención de Riesgos Laborales (SPRL) del HURC se encontró que en el año 2010 el $30.77 \%$ de los médicos residentes que adjudicaron una plaza de formación eran extranjeros, y el $87.5 \%$ eran iberoamericanos tal y como se aprecia en la Figura 1.

Asimismo entre los médicos residentes que provienen de países iberoamericanos apreciamos que la mayor procedencia para el año 2010 es de Venezuela 9 (33.33\%). Ver Figura 2. 
Figura 1. Médicos residentes extranjeros según lugar de procedencia

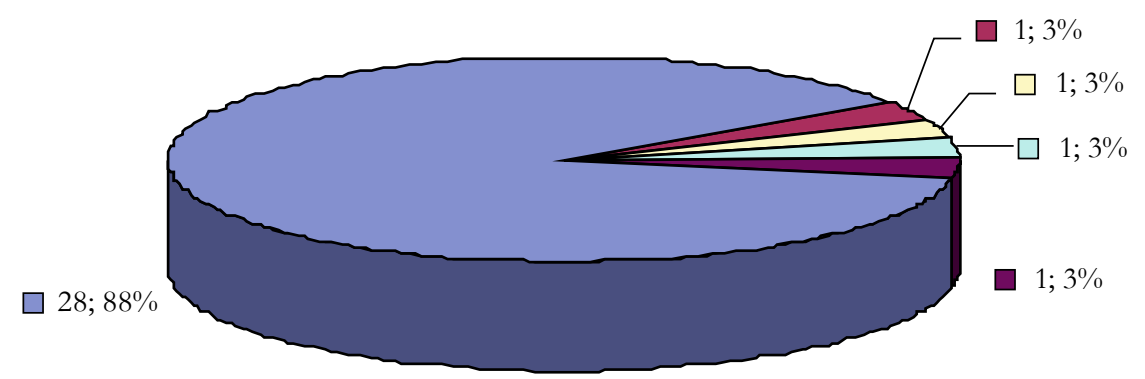

IBEROAMERICANOS $\square$ NIGERIA $\square$ RUMANIA $\square$ ALEMANIA $\square$ YIBUTI

Figura 2. Médicos residentes iberoamericanos según país de procedencia

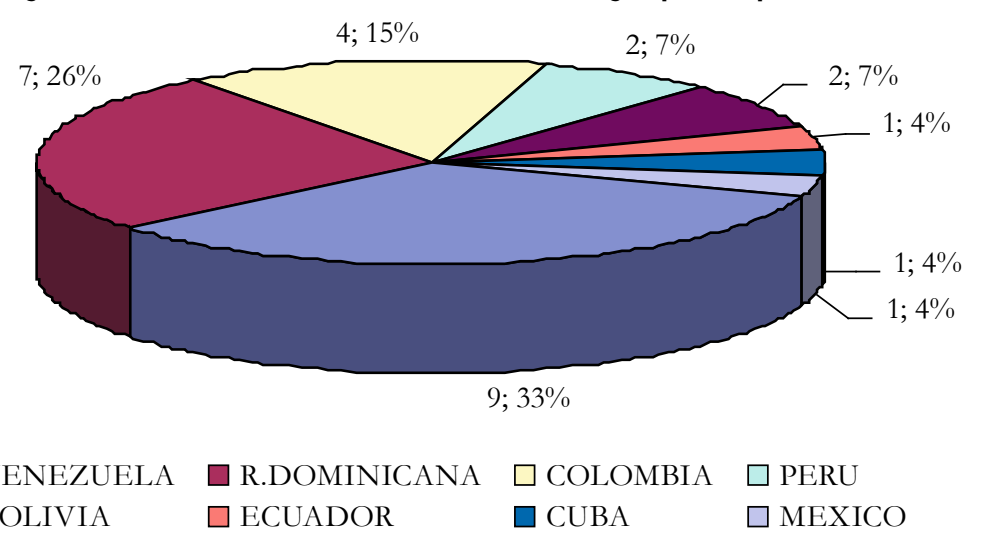

El SPRL del HURC anualmente a los médicos residentes de nueva incorporación se les realiza la Encuesta Inicial de Valoración de Salud y posteriormente analítica sanguínea basal, serología o antecedentes o cartilla de vacunación de parotiditis, rubéola, sarampión, varicela, hepatitis $\mathrm{B}$, hepatitis $\mathrm{C}$ y VIH para posteriormente pasar luego ser valorado por un médico del SPRL.

Debido a que el $30.77 \%$ de médicos residentes que ingresaron al Hospital Universitario Ramón y Cajal en el año 2010 eran extranjeros, es imprescindible que conozcamos su historial de vacunación y sobretodo si es que en sus respectivos países de procedencia cumplen o no con la pauta de vacunación recomendadas y en caso no la hayan cumplido debido a las políticas y normativas de sus respectivos países entonces actualizarla para el grupo de riesgo al cual pertenecen

Como se mencionó previamente el grupo de médicos residentes provenientes de iberoamérica era de $87.5 \%$, y debido a que la realidad de los países iberoamericanos es muy similar y además uno de los médicos residentes del SPRL es de nacionalidad peruana por lo que surge el interés por establecer pautas de vacunación a seguir en caso de que no se aporte documentación de vacunación previa para aquellos médicos residentes que provengan del Perú.

El objetivo de este estudio es realizar una revisión sobre la vacunación existente en el Perú, antecedentes y seroprevalencia de las patologías inmunoprevenibles en el personal sanitario; y en base a lo encontrado determinar las pautas de vacunación a seguir en caso no se aporte documentación de vacunación previa para aquellos médicos residentes de inicio que provengan del Perú. 


\section{MATERIAL Y MÉTODOS}

Se ha revisado la información correspondiente a pauta de vacunación en inmigrantes en territorio español.

Se hizo una búsqueda actualizada acerca de la vacunación en el Perú.

Se determinó el calendario de vacunación a seguir en caso no se aporte documentación de vacunación previa para aquellos médicos residentes de inicio que provengan del Perú.

\section{REVISIÓN BIBLIOGRÁFICA}

\section{Vacunación en inmigrantes}

Las recomendaciones de vacunación en la población inmigrante adulta son ${ }^{6,7,8}$ :

\section{Vacunación antitetánica y antidiftérica}

Si no hay antecedentes de vacunación antitetánica se precisan 3 dosis: 2 dosis con un intervalo mínimo de 4 semanas entre ellas y una tercera dosis con un intervalo mínimo de 6 meses tras la segunda.

En el caso de los adultos con primovacunación incompleta en ningún caso debe reiniciar la pauta de vacunación; se contabilizará cualquier dosis administrada previamente. Se completará la pauta de primovacunación hasta las 3 dosis. Los intervalos mínimos considerados son los mismos que en la pauta recomendada para adultos no vacunados.

Respecto a la dosis de recuerdo en adultos, para aquellos vacunados en la infancia correctamente según calendario vigente se recomienda una única dosis de recuerdo a los 65 años. Para aquellos vacunados en la infancia de manera incompleta se administrarán las dosis de recuerdo necesarias hasta alcanzar un total de 5 dosis (incluyendo la primovacunación con 3 dosis). Para aquellas personas adultas primovacunadas en la edad adulta con 3 dosis, se administrarán 2 dosis de recuerdo con un intervalo de 10 años entre dosis (el intervalo mínimo es de 12 meses) hasta completar un total de 5 dosis ${ }^{16}$.

Posteriormente se recomienda la administración de una única dosis de recuerdo en torno a los 65 años.

\section{Vacunación frente a la Hepatitis B}

La pauta de vacunación consiste en administrar 2 dosis con un mes de intervalo entre ellas y una tercera dosis que se puede administrar a las 8 semanas de la segunda, pero se recomienda que hayan pasado al menos 4 meses entre la primera y la tercera dosis.

Existe una pauta acelerada de 3 dosis con un mes de intervalo entre ellas pero la protección efectiva a largo plazo no se obtiene sin una cuarta dosis entre los 6 y 12 meses de la primera. Esta pauta puede utilizarse cuando exista un riesgo alto de transmisión y se precise una protección más rápida frente a la enfermedad.

La hepatitis B es endémica en muchos países por lo que se recomienda realizar marcadores previos a la vacunación (HbsAg, AntiHBs Ac y antiHBc Ac) en aquellas personas procedentes de países endémicos. El Perú es un país que tiene zonas endémicas de hepatitis $B$.

\section{Vacunación antipoliomielítica}

No se recomienda de forma rutinaria en las personas adultas españolas, pero se aconseja la vacunación de personas inmigrantes de países en los que todavía existe circulación del poliovirus salvaje (Afganistán, Bangladesh, Bhutan, Corea, Sri Lanka, 
Benín, Costa de Marfil, Egipto, Guinea, Chad, Burkina Fasso, Mali, Bostswana, Camerún, Togo, India, Indonesia, Irán, Myanmar, Nepal, Níger, Nigeria, Pakistán, República Centroafricana, Sudán, Tailandia) y de sus convivientes.

La primovacunación de personas de 18 ó más años se realizará siempre con Vacuna Antipoliomielítica Inactivada (VPI).

En adultos, la correcta vacunación antipoliomielítica consiste en la aplicación de tres dosis de VPI: dos dosis con un intervalo de al menos 1 mes entre ellas y la tercera dosis a los 6-12 meses de la segunda (con intervalo mínimo de 1 mes respecto a la segunda dosis).

\section{Vacunación con Triple Vírica}

La Región Europea de la Organización Mundial de la Salud (OMS) ha establecido un perfil de susceptibilidad frente al sarampión a alcanzar para conseguir la eliminación de la transmisión del sarampión en los países de la Región. Para alcanzar este perfil, las CCAA han establecido programas para, por un lado conocer el perfil de susceptibilidad en los diferentes grupos de edad y por otro, reforzar los programas de vacunación con actuaciones sobre los diferentes grupos para alcanzar este perfil propuesto por la OMS. En este contexto de eliminación del sarampión se debe realizar un esfuerzo adicional para evitar la reintroducción de virus y su posible diseminación. La procedencia de las personas inmigrantes, a veces de países con altas incidencias de sarampión, la posibilidad de viajes a sus países de origen, los antecedentes inciertos de vacunación y el hecho de que en muchos países la vacunación se realiza con el componente sarampión monovalente hace que el colectivo de inmigrantes pueda constituirse en una potencial bolsa de susceptibles capaz de originar brotes de las enfermedades incluidas en la vacuna triple vírica. Por otra parte es importante que las mujeres en edad fértil estén inmunizadas frente a la rubéola.

La población inmigrante adolescente debe recibir dos dosis de vacuna triple vírica, con un intervalo mínimo de un mes entre ellas.

Así mismo se recomienda que la población adulta reciba al menos 1 dosis de vacuna triple vírica siempre que no acrediten documentalmente la recepción previa de una dosis válida (administrada después de los 12 meses de edad) o el padecimiento previo o serología positiva frente al sarampión y paperas si se trata de varones y frente al sarampión y rubéola en el caso de las mujeres.

\section{Vacunación frente a Hepatitis A}

Esta es una enfermedad endémica en muchos países de los que provienen los inmigrantes, por lo que la mayoría de las personas adultas serán ya inmunes. Sin embargo y ante la posibilidad de que un inmigrante susceptible pueda adquirir la infección en un viaje a su país de procedencia se podría plantear la conveniencia de la determinación de anticuerpos y vacunación de los susceptibles cuando el inmigrante proceda de un país endémico (todos excepto Australia, Canadá, Estados Unidos, Europa Occidental, Japón y Nueva Zelanda).

Esta recomendación es especialmente importante en aquellos niños nacidos en España (o que han llegado a este país a una corta edad) de padres inmigrantes, cuando tengan previsto pasar una temporada en sus países de procedencia. Estos niños serán probablemente susceptibles a la enfermedad y deben ser vacunados antes del viaje.

Se vacunarán también aquellas personas pertenecientes a grupos de riesgo de esta enfermedad, independientemente de su condición de inmigrantes. Se recomienda la realización previa de anticuerpos de forma rutinaria si proceden de países endémicos.

La pauta de vacunación es de dos dosis con un intervalo mínimo de 6 meses entre ellas. 
Cuando se precise una vacunación conjunta frente a la hepatitis A y a la hepatitis B puede utilizarse la vacuna combinada $\left(\right.$ Twinrix $\left.^{\circledR}\right)$. En este caso, la pauta de vacunación será la de 0,1 y 6 meses.

\section{Otras vacunas}

Independientemente de su condición de inmigrantes y en función de la edad, pertenencia a otros grupos de riesgo y protocolos de cada CCAA, se administrarán el resto de las vacunas de acuerdo con sus indicaciones en la población adulta: vacuna antigripal (personas de 64 y más años y grupos de riesgo), antineumocócica (personas de 64 y más años si esta es la estrategia de vacunación en la CCAA y grupos de riesgo), antimeningocócica (dependiendo de los protocolos de vacunación en función de la edad de cada CCAA), Anti Hib (grupos de riesgo) y antivaricela (mayores de 10 años sin historia previa de enfermedad o de vacunación y grupos de riesgo de cualquier edad en las mismas circunstancias).

Además cuando estas personas vayan a realizar un viaje internacional se les aplicarán los protocolos establecidos de vacunación de viajeros en función del país que van a visitar, tipo de viaje, antecedentes de vacunación, etc.

\section{Vacunación en el Perú}

\section{Situación del aseguramiento en salud}

El sistema de salud de Perú está compuesto por cinco subsistemas que brindan servicios de salud a la población: a) el Ministerio de Salud (MINSA) que comprende al Seguro Integral de Salud (SIS) como un asegurador público con autonomía administrativa; b) La Seguridad Social en Salud - EsSaLud adscrito al Ministerio de Trabajo; c) las Sanidades de fuerzas Armadas (Marina, Aviación y Ejército) adscrita al Ministerio de Defensa; d) la Sanidad de la Policía Nacional del Perú (PNP) adscrita al Ministerio del Interior; y e) las instituciones del sector privado: entidades prestadoras de salud, aseguradoras privadas, clínicas y organizaciones de la sociedad civil (OSC).

El Estado ofrece servicios de salud para la población no asegurada, a través de los establecimientos de las regiones y, en el caso de Lima, del MINSA. El SIS provee servicios a la población en situación de pobreza y pobreza extrema a través de la red de establecimientos del MINSA. La sanidades de las fuerzas Armadas y Policiales cubren a los miembros de dichas corporaciones y sus dependientes.

EsSalud brinda servicios a los trabajadores formales y sus dependientes, así como a trabajadores informales a través de los seguros potestativos. Los servicios de EsSalud pueden ser complementados a través de las entidades prestadoras de salud (EPS), pertenecientes al sector privado. En el sector privado lucrativo existen clínicas para personas con capacidad de pago; otra parte del mercado privado ofrece servicios de medicina prepagada (compañías de seguros, autoseguros y seguros de las clínicas) para una pequeña proporción de la población. En el sector privado no lucrativo, existen instituciones de beneficencia que atienden a todo tipo de población. En este sistema de salud, actualmente, no existe equidad de acceso a los servicios ni a las prestaciones de salud.

Los afiliados a EsSalud cuentan con una cobertura "infinita", es decir, este seguro casi no tiene exclusiones en la práctica, en cambio todos los demás subsistemas de aseguramiento tienen planes limitados.

La cobertura del aseguramiento en salud ha ido aumentando en las últimas 2 décadas. En la Tabla II, se aprecia que en la década de 1980, 18\% de la población contaba con algún sistema de seguro. Esta cifra ha ido aumentando gracias a los esquemas de aseguramiento públicos, como el Seguro Escolar Gratuito y el Seguro Materno Infantil, que después se unificaron para conformar el SIS. En el año 2008, el SIS afilió a 18\% de la población. EsSalud, tradicionalmente el principal agente asegurador, cubre actualmente a 
$20 \%$ de la población total, mientras que el sector privado, incluyendo a las EPS, asume la limitada cobertura de menos de $4 \%$ de la población ${ }^{12}$.

Tabla II. Evolución del aseguramiento en el perú, 1985-2008

\begin{tabular}{lrrrrrrrrr}
\hline & $\mathbf{1 9 8 5}$ & $\mathbf{1 9 9 4}$ & $\mathbf{1 9 9 7}$ & $\mathbf{2 0 0 0}$ & $\mathbf{2 0 0 3}$ & $\mathbf{2 0 0 4}$ & $\mathbf{2 0 0 5}$ & $\mathbf{2 0 0 7}$ & $\mathbf{2 0 0 8}$ \\
\hline Con seguro & 17,6 & 23,4 & 22,7 & 32,3 & 41,7 & 36,5 & 35,3 & 43,3 & 42,0 \\
EsSalud & & 20 & 19,4 & 19,7 & 16,1 & 15,7 & 15,3 & 17,9 & 20,0 \\
SIS* & & & & & 24 & 16,6 & 16,3 & 18,4 & 18,0 \\
Otro** & 0 & 3,4 & 3,3 & 12,6 & 4,6 & 4,2 & 3,7 & 6,0 & 4,0 \\
Sin seguro & 82,4 & 76,6 & 77,3 & 67,7 & 58,3 & 63,5 & 64,7 & 56,7 & 58,0 \\
\hline
\end{tabular}

* SIS:Seguro Integral de Salud; En el año 2000, se incluye al Seguro Escolar Gratuito, luego transformado en SIS.

** En los años 2003-2005, en la categoría otro el seguro privado incluye a las EPS, seguros universitariosy el seguro escolar privado y las fuerzas Armadas y Policiales.

Los resultados del Censo Nacional de Población del año 2007, revelan que en el país, 43,3\% de la población cuenta con algún tipo de seguro de salud, es decir, 11 millones 599 mil 971 personas, no obstante, $57,7 \%$ de la población se encuentra aún desprotegida ${ }^{13}$.

Con la Ley 29344 del Aseguramiento Universal de Salud promulgada el 8 de abril del año 2009 se consigue que toda la población residente en el territorio nacional desde su nacimiento hasta su muerte cuente con derecho a la atención en salud con calidad y en forma oportuna.

\section{Sobre la vacunación}

El Programa de Inmunizaciones se empezó en el año 1964 con grandes campañas que dieron como resultado que en 1977 se declarara la eliminación del virus de la viruela en el mundo. Desde 1974 se empezó a trabajar en el programa de dosis ampliada, el cual tenía como meta lograr que los niños y niñas cumplieran con un esquema básico de vacunación contra el sarampión, poliomielitis, difteria, tétanos, tos convulsiva y tuberculosis. La meta era que todos los niños en el Perú estuviesen protegidos antes de cumplir el primer año de edad. En la década de los 90, luego de apoyar las grandes campañas de vacunación, UNICEF (Fondo Internacional de las Naciones Unidas de Auxilio a la Infancia) dio un impulso especial apoyando al Ministerio de Salud y a los servicios locales de salud en la educación de la población?

Antes de que existiera el programa de inmunizaciones el sarampión, la tos ferina y la difteria causaron la muerte de entre 1,500 y 2,500 niños al año. La poliomielitis fue también una de las principales causas de morbilidad crónica.

El Programa Ampliado de Inmunizaciones (PAI) como resultado de una acción conjunta de los países de América del Sur y de organismos internacionales, interesados en apoyar acciones tendientes a lograr coberturas universales de vacunación, con el fin de disminuir las tasas de mortalidad y morbilidad causadas por las enfermedades inmunoprevenibles y con fuerte compromiso de erradicar y controlar las mismas.

Las enfermedades objeto del Programa son: poliomielitis, sarampión, tétanos, difteria, tos ferina (pertussis), tuberculosis, rubéola y rubéola congénita, fiebre amarilla, Haemophilus influenzae, hepatitis $\mathrm{B}^{10}$.

Los logros del PAI en el Perú han sido progresivos y continuos. La estrategia de las Jornadas Nacionales de Vacunación realizadas a partir de 1985 permitió el incremento acelerado de las coberturas: en 1992 se superó el 80\% y en 1995 se lograron coberturas mayores al 90\%. Sin embargo, desde 1999 hasta el 2003 se observó un descenso progresivo en las coberturas de vacunación de aproximadamente uno o dos puntos por año.

En el año 1990 UNICEF y la OMS se plantearon una meta junto con los países del mundo: lograr la vacunación del $80 \%$. En todas partes del mundo, en cualquier grupo humano, por lo menos el $90 \%$ de los niños deberían tener las vacunas sarampión, polio, 
BCG y DPT. El Perú alcanzó solamente un 64\%. Cuando empezó el programa de vacunaciones en 1984, la cobertura era del 20\%: había centenares de casos de poliomielitis y de muertes por sarampión, y miles de casos de difteria y tétanos en los recién nacidos. Pero eso fue mejorando, y en el año 1994 se logró llegar al 80\%. También se realizaron campañas específicas para el control de enfermedades. Y en el año 1992 el Perú tuvo el último caso de poliomielitis en las Américas, en la localidad de Pichanaki, departamento de Junín. La poliomielitis es, desde entonces, una historia pasada en la medida que se siga vacunando a los niños.

En los últimos no han existido casos de sarampión en el Perú. Asimismo, se ha reducido mucho la incidencia de difteria, del tétanos y de la tos convulsiva, la cual sigue siendo un problema.

Las coberturas de vacunación son mayores en las zonas urbanas que en las rurales. Lamentablemente, existe un porcentaje de niños de las zonas rurales que no accede a los beneficios completos de la vacunación por problemas de accesibilidad. Todavía hay lugares donde no hay servicios, y si los hay, no tienen cadena de frío. No hay un acceso regular a las vacunas, y solo se puede acceder a esos lugares por brigadas que se hacen durante las jornadas de vacunación. Sin embargo, el Estado peruano, independientemente de los gobiernos, ha hecho un gran esfuerzo por mantener la prioridad en el tema de vacunación. Hoy el $100 \%$ de las vacunas que se usan para el programa regular de vacunación son compradas por el Estado?.

Las estrategias de erradicación de enfermedades han sido uno de los principales vehículos para desarrollar o mejorar las capacidades de vacunación y vigilancia en América del Sur, sentando las bases para el establecimiento de los programas actuales de vacunación. Algunas conquistas notables en materia de erradicación de enfermedades en la Región son la erradicación de la viruela y la poliomielitis y, en este momento, la interrupción de la transmisión autóctona del sarampión.

Se conoce que para el caso de Perú la polio se erradicó en 1991, el sarampión en 2001 y la rubéola en el año $2006^{11}$.

\section{Vacunación en el personal sanitario}

El Reglamento de Seguridad y Salud en el Trabajo ${ }^{15}$, mediante Decreto Supremo No 009-2005 establece el principio de prevención en la cual el empleador asumirá las implicancias económicas, legales y de cualquiera otra índole, como consecuencia de un accidente o enfermedad que sufra el trabajador en el desempeño de sus funciones o a consecuencia de él, conforme a las normas vigentes; asimismo el empleador debe tomar las medidas de prevención y protección.

De acuerdo a lo referido previamente con la finalidad de disminuir el riesgo biológico en el personal sanitario, una de las medidas más eficaces es la vacunación.

Actualmente en el Perú la normativa sobre el esquema de vacunación está basada en la resolución Ministerial 457-2009 del Ministerio de Salud en el que se aprueba la norma técnica de salud que establece el esquema nacional de vacunación que es aplicable en todos los establecimientos públicos y privados del sector salud ${ }^{14}$. En dicho esquema de vacunación podemos apreciar que se incluye también al personal sanitario y se les pauta vacunación contra hepatitis $\mathrm{B}$, Influenza, fiebre amarilla y Difteria-Tétanos.

Cabe mencionar que tanto en las universidades donde se forma al personal sanitario ni en los hospitales donde trabaja dicho personal existe un Servicio de Prevención de Riesgos Laborales por lo que no podemos determinar si ésta población en riesgo ha sido vacunada anteriormente a la aplicación de la normativa de vacunación del año 2009. 


\section{DISCUSIÓN Y CONCLUSIONES}

- La estrategia de vacunación en el Perú ha tenido grandes altibajos respecto a la cobertura en la población; si bien la última normativa de vacunación es más extensa y pretende abarcar todos los establecimientos públicos y privados del sector salud sólo tiene 1 año de vigencia con lo cual no se puede determinar que los médicos residentes procedentes de Perú tengan una cobertura de vacunación adecuada.

- No existen datos publicados sobre seroprevalencia de enfermedades inmunoprevenibles en la literatura peruana por lo que no podemos determinar si la población está o no inmunizada y en base a ello tomar una actitud sobre vacunación.

- De acuerdo a la información obtenida sobre vacunación en el Perú podemos concluir que no existe evidencia que nos indique que existe una correcta pauta de vacunación en los médicos residentes que proceden de Perú, por tanto si no se nos aporta la cartilla de vacunación correspondiente se procederá a la vacunación como si fuese un adulto no vacunado (ver Tabla III), siempre teniendo en cuenta que si existe documentación de dosis previas se completarán las pautas sin reiniciar o repetir las dosis.

Tabla III. Calendario de vacunación a seguir en médicos residentes procedentes de Perú que no aporten cartilla de vacunación

\begin{tabular}{llll}
\hline \multicolumn{1}{c}{ Vacuna } & & Calendario (mes) \\
\cline { 2 - 4 } & $\mathbf{0}$ & $\mathbf{1}$ & $\mathbf{6}$ \\
\hline Tripe vírica $^{1}$ & $\mathrm{X}$ & $\mathrm{X}$ & $\mathrm{X}^{2}$ \\
Difteria-tétanos & $\mathrm{X}$ & $\mathrm{X}$ & $\mathrm{X}^{2}$ \\
VPI (poliomielitis) & $\mathrm{X}$ & $\mathrm{X}$ & $\mathrm{X}$ \\
Hepatitis B & $\mathrm{X}$ & $\mathrm{X}^{2}$ \\
Hepatitis A & & \\
\hline 1. Una o 2 dosis en función de la edad. & $\mathrm{X}$ & & \\
2. La tercera dosis se puede administrar entre los 6-12 meses de la segunda & & \\
3. El número máximo de dosis es 6. & & \\
4. Recomendado en residentes que trabajen en laboratorios. & & \\
\hline
\end{tabular}

\section{REFERENCIAS BIBLIOGRÁFICAS}

1. Farjas M. Vacunación en inmigrantes. Fisterra. España [en línea] <http://www.fisterra.com/vacunas/ pdf/Inmigrante.pdf $>$ [Consulta: 18 octubre 2010].

2. Gil de Miguel A. Vacunación en inmigrantes. Calendarios incompletos. Actualización en vacunas 2009; 43-49.

3. Serrano C, Jiménez L, Fernández-Pacheco B, et al. Seroprevalencia y conocimiento de vacunación en nuevos residentes. Med Segur Trab 2009; 55 (214): 101-110.

4. Centers for Disease Control and Prevention. Immunization of Health-Care Workers: recommendations of the Advisory Committee on Immunization Practices (ACIP) and the Hospital Infection Control Practices Advisory Committee (HICPAC). MMWR 1997;46(No. RR-18).

5. Diario El País. [En línea]. Disponible en Web: http://www.elpais.com/articulo/sociedad/45/nuevos/MIR/ extranjeros/elpepusoc/20100405elpepusoc_3/Tes [Consulta: 06 de octubre de 2010]

6. American Academy of Family Physicians. AAFP Clinical Recommendations for Immunizations. [Internet]. 2009. Disponible en: http://www. aafp.org/x10631.xml

7. Consejería de Sanidad y Consumo de la Región de Murcia. Programa de vacunación para inmigrantes. [Internet]. Mursia Salud; 2002. [acceso 18/10/2010]. Disponible en: http://www.murciasalud.es/pagina. php?id=595\&idsec $=85$ 
8. Recommended Adult Immunization Schedule- United States. MMWR October 13, 2006 / 55(40);Q1-Q4 [Texto completo]

9. Diagnóstico Perú. Unicef. Disponible en Web: http://www.unicef.org/peru/_files/notas_prensa/ carpetasinformativas/vacunacion.pdf [Consulta: 19 de octubre de 2010]

10. Programa Ampliado de Inmunizaciones. Publicaciones OPS. Disponible en Web: http://www.paho.org/ Spanish/DD/PUB/PAI.pdf [Consulta: 19 de octubre de 2010]

11. Agencia Peruana de Noticias, Lima 7 de julio del 2007. Disponible en Web: http://www.andina.com.pe/ espanol/Noticia.aspx?id=u5PIY29h5HA= [Consulta: 19 de octubre de 2010]

12. Wilson L, Velásquez A, Ponce C. La ley marco de aseguramiento universal en salud en el Perú: análisis de beneficios y sistemartización del proceso desde su concepción hasta su promulgación. Rev Peru Med Exp salud Publica. 2009; 26(2):207-17.

13. Instituto Nacional de Estadística e Informática. 10. Perú: Crecimiento y distribución de la población, 2007. Censos nacionales 2007: XI de población y VI de vivienda. Primeros resultados. Lima: INEI; 2008.

14. Ministerio de Salud del Perú. Norma Técnica de Salud que establece el Esquema Nacional de Vacunación. Lima 9 de julio del 2009 (RM 457-2009/MINSA).

15. Ministerio de Trabajo y Promoción de Empleo del Perú. Reglamento de Seguridad y Salud en el Trabajo. Decreto Supremo No 009-2005-TR. Lima 29 de septiembre del 2005.

16. Ministerio de Sanidad y Consumo. Vacunación en Adultos. Recomendaciones: vacuna de difteria y tétanos. Actualización 2009. Documentación aprobada por la Comisión de Salud Pública, con fecha 18 de febrero del 2009.

||||||||||||||||||||||||||||||||||||||||||||||||||||||||||||||||||||||||||||||||||||||||||||||||||||||||||||||||||||||||||||||||||||||||||||||||||||||||||||||||||||||||||||||||||||||||||||||||||||||||||||||||||| 